$\mathbb{P}$ periodica polytechnica

Civil Engineering

57/2 (2013) 123,128

doi: 10.3311/PPci.7168

http://periodicapolytechnica.org/ci

Creative Commons Attribution (1)

RESEARCH ARTICLE

\section{Dynamic stability analysis of a thin-walled beam subjected to a time periodic gradient bending moment}

\author{
Abbas Talimian / Gábor M. Vörös
}

Received 2012-04-16, revised 2012-12-01, accepted 2013-05-31

\begin{abstract}
This paper analyzes the dynamic stability of a thin walled beam subjected to non-uniform bending moment. It provides the detail of study the influence of bending moment gradient on instability regions. The second order rotation effect is considered for performing a correct flexural-torsional analysis. The analysis is based on the potential energy principle and adopting the Ritz method. Matrix form of Mathieu-Hill type equation is governed to analyze the stability problem. The paper presents Bolotin's approximations on periodic excitation leads to the stability regions of the structure. Relevant graphs are presented for different loading parameters. Ritz method's terms number and bending moment gradient's coefficient are discussed in detail as well.
\end{abstract}

\section{Keywords}

dynamic stability · buckling · Mathieu-Hill equation · instability regions $\cdot$ thin walled beams

\section{Abbas Talimian}

Department of Vehicle Elements and Vehicle Structure Analysis, Budapest University of Technology and Economics, H-1521 Budapest, Múegyetem rkp.3, Hungary

e-mail: talimian@kme.bme.hu

\section{Gábor M. Vörös}

Department of Vehicle Elements and Vehicle Structure Analysis, Budapest University of Technology and Economics, H-1521 Budapest, Múegyetem rkp.3, Hungary

\section{Introduction}

Thin walled beams are used commonly in vehicle industry as a part of chassis or frame structures. Different methods have been suggested for analyzing these structures' behavior and their stability analysis by researchers.For instance following papers can be mentioned among lots of publications in this field. Dokumaci expressed an exact analytical expression for solving the bending-torsion problems in 1987 [1]. This Result was expanded by Bishop et al. after two years to cover up the torsionwarping effect which is an important point for thin walled beams [2]. Pi and Bradford showed the approximations effects on an open thin-walled cross-section in 2001 [3]. Vörös investigated coupling of bending and torsional vibrations due to initial load [5]. Vibrational response of a beam, affected by an initial bending moment load was searched by Vörös and Talimian [6].

Considering a dynamic instability problem induced by periodic excitation leads to the well known Mathieu-Hill equation [15]. Faraday was one of the pioneers in discovering parametric resonance [7]. N.M. Beliaev worked on the Stability of prismatic rods in 1924 [8]. The stability of dynamic systems was explained by V.V. Bolotin in 1962 [9]. Brown et al. brought finite element technique for inspecting the dynamic stability behavior into play [10]. S.P. Timoshenko and J.M Gere published a literature on theory of elastic stability. In 1975, Thomas and Abbas worked on shear deformation and rotary inertia effects on stability [11]. Snehasis Ganguly and Datta looked over the second regions of a uniform beam dynamic stability [12]. C.E. Majorana et al. in 1998 expand a finite approach for stability of frames and beams [13].

Uniform initial moment loading effect on a thin walled beam was shown in [5]. However, the question which was left open to our best knowledge is the stability of a thin walled-beam subjected to periodic initial moment loading. The present research's goal is to find the instability domains for a thin-walled beam which has fork like supports that prevent torsional rotation and allow free warping at each end, and is loaded by timedependent bending moment initially. For this propose motion's equation were derived by help of potential energy [6]. Using Ritz-Galerkin as a common finite element technique guides to 
matrix form of Mathieu-Hill equation such as given in [11]. By solving the latter system of equations, the stable and unstable areas will be involved.

\section{Formulations}

\subsection{Virtual work principal}

Basic assumptions used for deriving present study's equations are: beam is straight and prismatic, Euler-Bernoulli bending and Vlasov's torsion theory, rotations are large, strains are small, and material is homogenous, isotropic and linearly elastic. The axis $x$ is the beam's axis and $y, z$ axes are in the section plane and coincide with principal axes' of the cross section. The centroid and shear centre are signed by $\mathrm{C}$ and $\mathrm{S}$ respectively and the external loads are applied at point $\mathrm{P}$. The geometrical eccentricity (distance between centroid and shear center) is represented by e, which will be zero for symmetric cross section. External loads are applied along points $\mathrm{P}$ located $\mathrm{h}$ from the shear center S. (Fig. 11).

A beam supported by fork like supports with unequal end moments $M$ and $\psi M$ is given in Fig. 1 1 a. The bending moment and shear force distribution in the beam are ${ }^{0} M_{y}$ and ${ }^{0} V_{z}$ respectively, can be expressed as follows [6]:

$$
{ }^{0} M_{y}=-M\left(1-x \frac{1+\psi}{L}\right), \quad{ }^{0} V_{z}={ }^{0} M_{y}^{\prime}=M \frac{1+\psi}{L}
$$

Coupled equations with variable coefficients were derived by applying virtual work principle, while strains are supposed to be small and rotations are large [4,5] and [6]. The virtual work principle is [6]:

$$
\delta \frac{1}{2} \int_{0}^{L}\left[E I_{z}\left(v^{\prime \prime}\right)^{2}+E I_{\omega}\left(\alpha^{\prime \prime}\right)^{2}+\left(G J+{ }^{0} M_{y} \beta_{y}\right)\left(\alpha^{\prime}\right)^{2}+2^{0} M_{y} v^{\prime \prime} \alpha\right] d x
$$$$
+\int_{0}^{L} \rho\left[A(\ddot{v}+e \ddot{\alpha}) \delta v+\left(A e \ddot{v}+I_{p s} \ddot{\alpha}\right) \delta \alpha\right] d x-M \delta\left[v^{\prime} \alpha\right]_{0}
$$$$
-M \psi \delta\left[v^{\prime} \alpha\right]_{L}+\operatorname{Rt} \delta\left[\frac{\alpha^{2}}{2}\right]_{0}^{L}=0
$$

In equation (2), $v(x, t)$ is the rigid body translation of the section in the $y$ direction. Rotation about the shear centre is given by $\alpha(x, t) . E$ and $G$ are elastic and shear module, respectively. $M$ and $R$ are static moment and force reaction of the structure, Fig. 117. The cross sectional area is nominated by $A$. $I_{z}$ is moment of inertia respect to vertical axes. $I_{\omega}$ is warping constant respect to shear center [5].

The prime and dot denotes differentiation with respect to $x$ and $t$ as longitudinal and time variable. It has to be mentioned that energy functional (2) was consistently obtained corresponding to semi tangential internal moments. The detailed derivation may be found in Kim et al. [14] and Vörös [4]. Having the boundary conditions, Fig. 1p, and simplifies assumption $\left({ }^{0} M_{y} \beta_{y}=0\right), 2$ will be rearranged as:

$$
\begin{aligned}
& \delta \frac{1}{2} \int_{0}^{L}\left(E I_{z}\left(v^{\prime \prime}\right)^{2}+E I_{\omega}\left(\alpha^{\prime \prime}\right)^{2}+G J\left(\alpha^{\prime}\right)^{2}+2^{0} M_{y} v^{\prime \prime} \alpha\right) d x \\
& +\int_{0}^{L} \rho\left(A \ddot{v} \delta v+I_{p s} \ddot{\alpha} \delta \alpha\right) d x+\int_{0}^{L} \rho A e(\ddot{\alpha} \delta v+\ddot{v} \delta \alpha) d x
\end{aligned}
$$

\subsection{Finite degrees model}

For having an approximate solution of (3) and latterly evaluating stability regions of the structure, the well-known RitzGalerkin method shall be expanded as a finite degrees of freedom model. In this sense an approximated functions are needed for the lateral displacement and torsional rotation, which can be such:

$$
v(x, t)=\sum_{i=1}^{n} F_{i}(t) f_{i}(x), \quad \alpha(x, t)=\sum_{i=1}^{m} V_{i}(t) g_{i}(x)
$$

Due to Ritz-Galerkin approximation solution the geometrical boundary condition, where mentioned in Fig. 1.b) has to be satisfied completely by selecting apt functions in (4). A usual possible assumption for a beam with fork like supports which prevent torsional rotation and allow free warping as shown in Fig. 1.b) can be:

$$
f_{i}(x)=\sin \left(\frac{i \pi x}{L}\right), \quad g_{i}(x)=\sin \left(\frac{i \pi x}{L}\right)
$$

Here it has worth to be mentioned for uniform bending, $\psi=$ -1 , the beam buckles with the half-sine wave length, while the lateral deflected shape is an anti-symmetrical full sine for asymmetric bending, $\psi=1$. Torsinal shape $\alpha(x, t)$ is a symmetrical half-sine form. The minimum number of terms should be $n=1$, $m=2$ [6] to cover up the solution while $\psi$ is varying from minus one (uniform bending) to one (asymmetric bending). Substituting (5) and (4) into (3), and applying the increment yields:

$$
\begin{aligned}
& \sum_{i=1}^{n} \sum_{j=1}^{n}\left(E I_{z} F_{i}(t) A_{i j} \delta F_{j}(t)+\rho A \ddot{F}_{i}(t) E_{i j} \delta F_{j}(t)\right) \\
& +\sum_{i=1}^{m} \sum_{j=1}^{m}\left(E I_{\omega} V_{i}(t) B_{i j} \delta V_{j}(t)+G J V_{i}(t) C_{i j} \delta V_{j}(t)\right. \\
& \left.\quad+\rho I_{p s} \ddot{V}_{i}(t) H_{i j} \delta V_{j}(t)\right) \\
& +\sum_{i=1}^{n} \sum_{j=1}^{m}\left(\delta F_{i}(t) D_{i j} V_{j}(t)+F_{i}(t) D_{i j} \delta V_{j}(t)\right. \\
& \left.\quad+\rho A e \ddot{F}_{i}(t) H_{i j}^{*} \delta V_{j}(t)\right) \\
& +\sum_{i=1}^{m} \sum_{j=1}^{n} \rho A e \ddot{V}_{i}(t) E_{i j}^{*} \delta F_{j}(t)=0 .
\end{aligned}
$$

Here in (6) $\mathbf{A}-\mathbf{H}$ are numerical matrices, which are given in details in the appendix. Defining two vectors that their elements are unknown time dependent functions of (4) such as:

$$
\mathbf{F}(t)=\left[F_{1}(t) \cdots F_{n}(t)\right]^{T}, \quad \mathbf{V}(t)=\left[V_{1}(t) \cdots V_{m}(t)\right]^{T}
$$




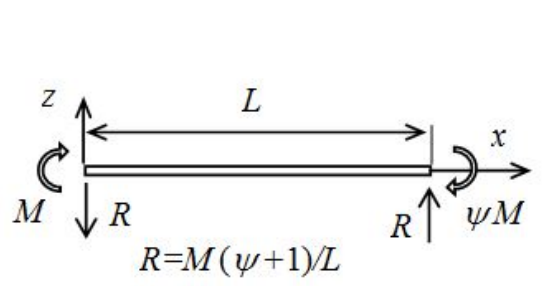

(a)

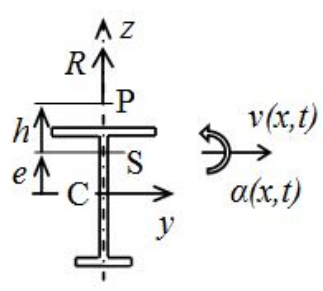

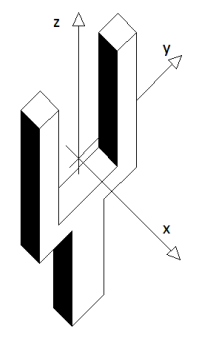

$v(0, t)=\alpha(0, t)=0$

$v(L, t)=\alpha(L, t)=0$

$v^{\prime \prime}(0, t)=\alpha^{\prime \prime}(0, t)=0$

$v^{\prime \prime}(L, t)=\alpha^{\prime \prime}(L, t)=0$

(b)

Fig. 1. Cross section reaction forces (a), fork like support's boundary conditions(b)

Help to reformulate 6 in the following compressed form,

$$
\begin{aligned}
& E I_{z} \mathbf{F}^{T} \mathbf{A} \delta \mathbf{F}+E I_{\omega} \mathbf{V}^{T} \mathbf{B} \delta \mathbf{V}+G J \mathbf{V}^{T} \mathbf{C} \delta \mathbf{V}+\delta \mathbf{F}^{T} \mathbf{D V}+\mathbf{F}^{T} \mathbf{D} \delta \mathbf{V} \\
& +\rho A \ddot{\mathbf{F}}^{T} \mathbf{E} \delta \mathbf{F}+\rho A e \ddot{\mathbf{V}}^{T} \mathbf{E}^{*} \delta \mathbf{F}+\rho I_{p s} \ddot{\mathbf{V}}^{T} \mathbf{H} \delta \mathbf{V}+\rho A e \ddot{\mathbf{F}}^{T} \mathbf{H}^{*} \delta \mathbf{V}=0
\end{aligned}
$$

For having a matrix form, it is necessary to define a new vector $\mathbf{X}$

$$
\mathbf{X}=\left[F_{1}(t) \cdots F_{n}(t) V_{1}(t) \cdots V_{m}(t)\right]^{T}=\left[x_{1} \cdots x_{n+m}\right]^{T}
$$

Matrix form of (8) is as following equation, which $\mathbf{M}, \mathbf{K}_{e}$ and $\mathbf{S}$ are mass, elastic stiffness and stability matrices respectively such as given in the appendix.

$$
\mathbf{M} \ddot{\mathbf{X}}+\left(\mathbf{K}_{e}+M(t) \mathbf{S}\right) \mathbf{X}=\mathbf{0}
$$

\section{Periodic excitation}

There exist no analytical solution for second order differential equation with periodic coefficient, although different solutions' methods have been explored in this type. A beam which is analyzed in this paper subjected to a concentrated periodical moment at its ends by the following given function.

$$
M(t)=M_{s}+M_{d} \cos (\Omega t)
$$

Here $M_{s}$ and $M_{d}$ are static and dynamic amplitude of time dependent moment respectively. The excitation frequency is designated by $\Omega$. Introducing $\lambda$ and $\mu$ as a static and dynamic buckling moment respectively (12) the initial moment distribution (11) will be (13):

$$
\begin{gathered}
\lambda=\frac{M_{s}}{M_{c r 0}}, \quad \mu=\frac{M_{d}}{M_{c r} 0} \\
M=M_{c r 0}(\lambda+\mu \cos (\Omega t))
\end{gathered}
$$

$M_{c r 0}$ is The fundamental static buckling moment when moment distribution factor, $\psi$, set to minus one [6]. Replacing [13] into (10) returns the final matrix form of motion's equation:

$$
\mathbf{M} \ddot{\mathbf{X}}+\left(\mathbf{K}_{e}+M_{c r 0}(\lambda+\mu \cos (\Omega t)) \mathbf{S}\right) \mathbf{X}=\mathbf{0}
$$

which is named Mathieu-Hill. A few methods have been suggested for solving Mathieu-Hill equation, as experimental solutions; Bolotin monograph, Galerkin , Lyapunov's second method, asymptotic techniques and perturbation and iteration have been introduced as well. In the current research, a solution based on Brown [10] with $2 T$ period (first approximation of first region of stability) was applied for (14). An approximated periodic solution, which is advised for [14], is [12,15]:

$$
\mathbf{X}=\sum_{k=1,3,5}^{\infty} \mathbf{a}_{\mathbf{k}} \sin \left(\frac{k \Omega t}{2}\right)+\mathbf{b}_{\mathbf{k}} \cos \left(\frac{k \Omega t}{2}\right)
$$

By substituting (15) in (14):

$$
\left(\mathbf{K}_{e}+M_{c r 0}(\lambda+\mu \cos (\Omega t)) \mathbf{S}-\frac{\Omega^{2}}{4} \mathbf{M}\right) \mathbf{X}=\mathbf{0}
$$

Linear independence of trigonometric functions were introduced in $15, \sin \left(\frac{\Omega t}{2}\right), \sin \left(\frac{3 \Omega t}{2}\right), \cdots$ and $\cos \left(\frac{\Omega t}{2}\right), \cos \left(\frac{3 \Omega t}{2}\right), \cdots$ yields (16) can be separated regarding to sine and cosine terms. Homogeneous linear algebraic system of equations respect to trigonometric functions, $\mathbf{a}_{k}$ and $\mathbf{b}_{k}$ are derived, hence for having non-trivial solution the coefficient matrices determinant for each matrix equation has to be set to zero. As far as (15) contains infinite terms, in this survey the first component of each matrix were selected only as the first approximation. The stability curves (borders) are derived from latter matrices' determinant, had to be equal to zero:

$$
\begin{aligned}
& \operatorname{det}\left(-\left(\frac{\Omega}{2}\right)^{2} \mathbf{M}+\mathbf{K}_{e}+M_{c r 0}\left(\lambda+\frac{\mu}{2}\right) \mathbf{S}\right)=0 \\
& \operatorname{det}\left(-\left(\frac{\Omega}{2}\right)^{2} \mathbf{M}+\mathbf{K}_{e}+M_{c r 0}\left(\lambda-\frac{\mu}{2}\right) \mathbf{S}\right)=0
\end{aligned}
$$

\section{Numerical example}

There are two necessary terms, should be evaluated before drawing the first approximated stability borders of first region of stability; fundamental natural bending frequency and static buckling moment as a fraction of the first approximation of the fundamental buckling moment. By substituting $\lambda=0$ and $\mu=$ 0 in (16), the Eigen-values problem will represent the natural frequencies (free vibration). Two lateral bending and a torsional frequency were nominated as fundamental natural frequencies [6]. Furthermore finding critical static buckling moment can be done similarly with assuming $\lambda=1$ and $\mu=0$. Incidentally, these values will be as follows for the given data in Fig. [2 [6].

$$
\omega=275.46[1 / \mathrm{s}], \quad M_{c r 0}=91 \mathrm{kNm}
$$


It is worth to mention that the frequency in 19 is the first lateral bending i.e. the minimum frequency of the beam as well.

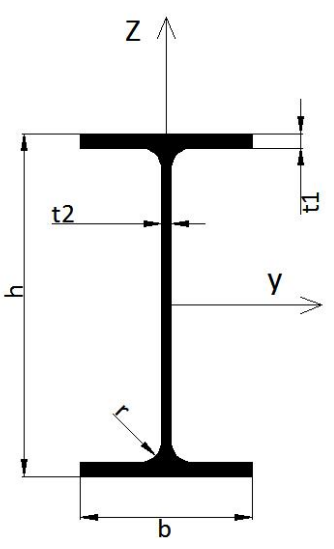

$\begin{array}{lll}h[\mathrm{~mm}]=200 & r[\mathrm{~mm}]=12 & I_{z}\left[\mathrm{~mm}^{4}\right]=1423683 \\ b[\mathrm{~mm}]=100 & L[\mathrm{~mm}]=2000 & J\left[\mathrm{~mm}^{4}\right]=68464 \\ t_{1}[\mathrm{~mm}]=8.5 & A\left[\mathrm{~mm}^{2}\right]=2848 & I_{\omega}\left[\mathrm{mm}^{6}\right]=12746157814 \\ t_{2}[\mathrm{~mm}]=5.6 & I_{y}\left[\mathrm{~mm}^{4}\right]=19431689 & I_{p S}\left[\mathrm{~mm}^{4}\right]=20855372\end{array}$

Fig. 2. Section properties of IPE200

Taking equations (17), (18) and (19) into account, and plotting the characteristic equations implicitly for a given cross section in Fig. 2 illustrates a graph as Fig. 3 It shows two different areas. Zones where are outside curves, are nominated stable because of a bounded response of structure to the periodic load. Inside curves' area illustrates the region which was loading parameters value causes instability. Fig. 3 shows only the first stability region of first stability approximation analysis, which is the most important one, among infinite number of graphs depending on the number of selected terms in (15). First the optimum number of terms should be defined in the Ritz method. Fig. 3 shows the effect of increasing terms on the border curves for the selected parameter of $n, m=\{1,2,3\}$ for two types of loading; while $\psi=-1$ for the uniform bending and $\psi=1$ for the asymmetric one. Increasing terms number has no obvious effect on the accuracy of the first approximation. Setting $n=1$ and $m=2$ in (4) is enough for achieving proper results. The non-dimensional ratio of excitation frequency over first bending fundamental natural frequency was used to simplify the plots. This factor is given by $\varphi=\Omega / \omega_{b 1}$.

Subsequent graphs (Figs. 4 and 5) show the critical static buckling moment percentage $(\lambda)$ effect on the stability regions. Graphs are given for $\psi=-1$ (uniform bending) and $\psi=1$ (asymmetric bending) as two boundary of loading values. As it can be seen on the plots enlarging the share of static buckling moment percentage, causes the instability border line being further from each other which is deduced as an increasing in the structure's instability.

The stability borders plotted in Fig. 3 by employing (17), (18) are approaching toward each other by changing the moment distribution factor, $\psi$, between minus one and one (Table 1 and Fig. 6. It means that for asymmetric loading, in the range of first approximation stability the beam is always stable.

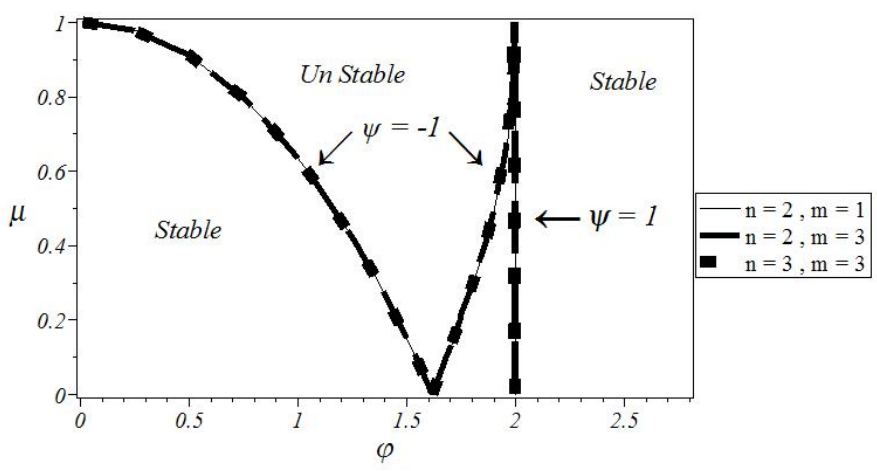

Fig. 3. Comparison of solutions for different Ritz terms 44, $\lambda=0.5$ and $e=0$

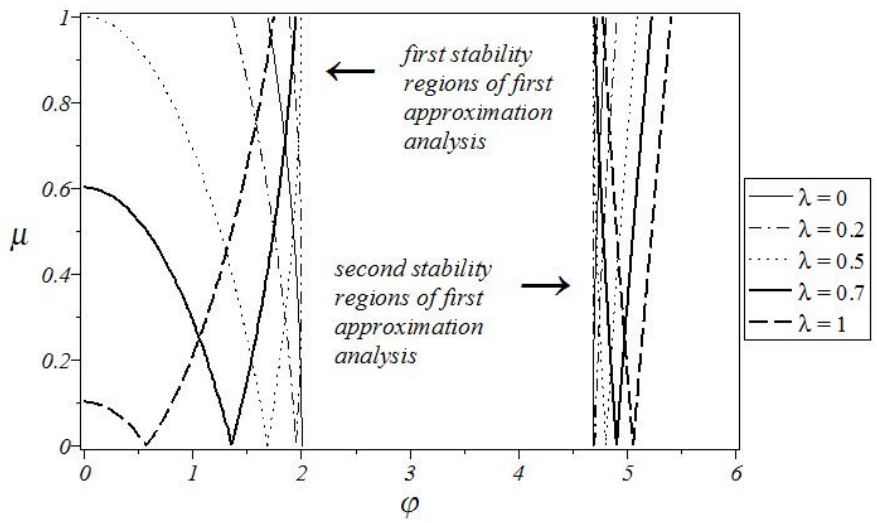

Fig. 4. Instability regions for different values of $\lambda$ while $\psi=-1$ and $e=0$

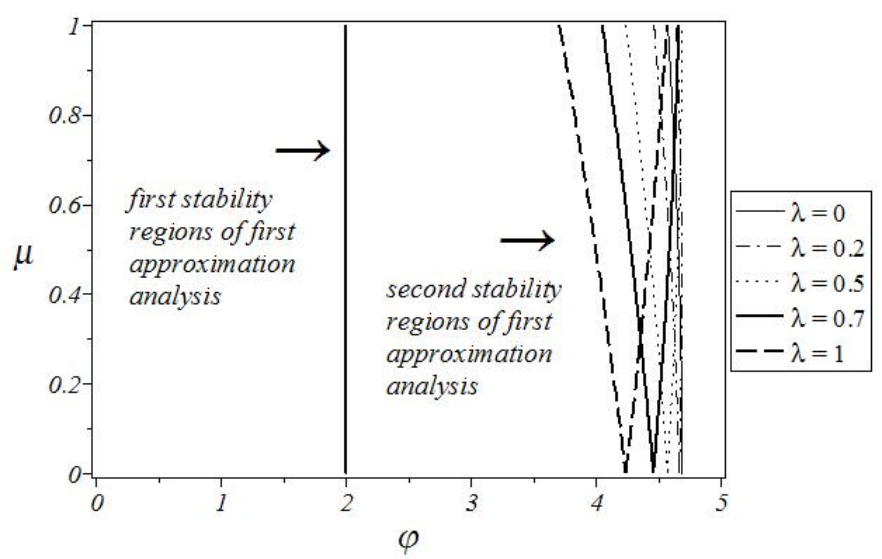

Fig. 5. Instability regions for different values of $\lambda$ while $\psi=1$ and $e=0$ 
Tab. 1. Changing $\varphi$ versus $\psi=[-1,1]$, while $e=0$ and $\lambda=0.5$

\begin{tabular}{ccccccc}
\hline & \multicolumn{2}{c}{$\mu=0.2$} & \multicolumn{3}{c}{$\mu=0.5$} & \multicolumn{2}{c}{$\mu=0.9$} \\
$\psi$ & Eq. 17 & Eq. 18 & Eq. 17 & Eq. 18 & Eq. 17 & Eq. 18 \\
\hline-1.00 & 1.46 & 1.75 & 1.17 & 1.90 & 0.53 & 2.00 \\
\hline-0.87 & 1.52 & 1.78 & 1.27 & 1.91 & 0.79 & 2.00 \\
\hline-0.73 & 1.59 & 1.81 & 1.37 & 1.92 & 0.98 & 2.00 \\
\hline-0.60 & 1.64 & 1.83 & 1.46 & 1.93 & 1.14 & 2.00 \\
\hline-0.47 & 1.69 & 1.86 & 1.54 & 1.94 & 1.27 & 2.00 \\
\hline-0.33 & 1.74 & 1.88 & 1.61 & 1.95 & 1.39 & 2.00 \\
\hline-0.20 & 1.79 & 1.90 & 1.68 & 1.96 & 1.50 & 2.00 \\
\hline-0.07 & 1.83 & 1.92 & 1.74 & 1.97 & 1.59 & 2.00 \\
\hline 0.07 & 1.87 & 1.94 & 1.80 & 1.98 & 1.68 & 2.00 \\
\hline 0.20 & 1.90 & 1.96 & 1.85 & 1.98 & 1.75 & 2.00 \\
\hline 0.33 & 1.93 & 1.97 & 1.89 & 1.99 & 1.82 & 2.00 \\
\hline 0.47 & 1.95 & 1.98 & 1.93 & 1.99 & 1.88 & 2.00 \\
\hline 0.60 & 1.97 & 1.99 & 1.96 & 2.00 & 1.93 & 2.00 \\
\hline 0.73 & 1.99 & 2.00 & 1.98 & 2.00 & 1.97 & 2.00 \\
\hline 0.87 & 2.00 & 2.00 & 2.00 & 2.00 & 1.99 & 2.00 \\
\hline 1.00 & 2.00 & 2.00 & 2.00 & 2.00 & 2.00 & 2.00 \\
\hline & & & & & &
\end{tabular}

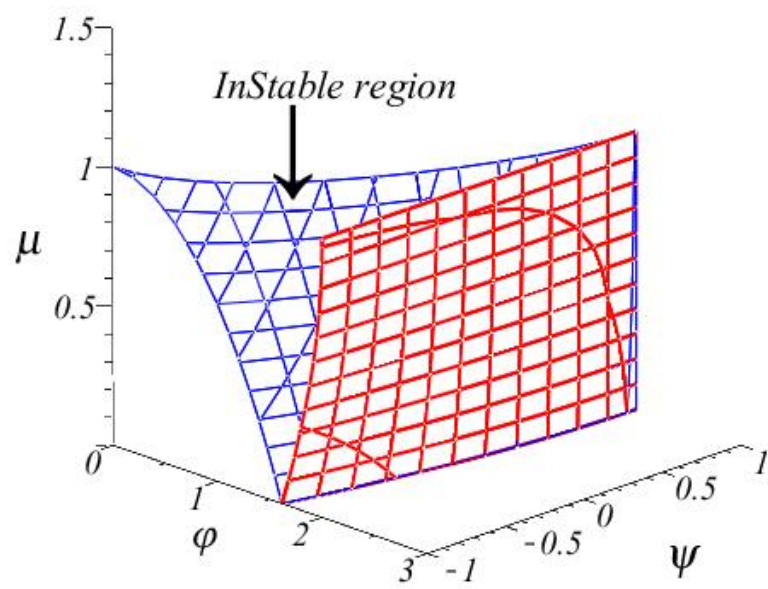

Fig. 6. Instability regions for different values for $\psi=[-1,1]$, while $e=0$ and $\lambda=0.5$ 


\section{Conclusions}

Present research focused on the analysis of the non-uniform time dependent initial moment for a thin-walled beam element with fork like supports. Applying Valsov theory in warping displacement yielded, a coupling in motion equation derived from virtual work principle. Mathieu-Hill equation and first approximated stability borders of first region of stability were adopted as well. Main results of the survey can be stressed as:

- As it can be seen in Fig. 3 having higher terms in Ritz approximation will have no magnificent effect on the regions of stability in first approximated stability analysis.

- Enlarging static buckling moment percentage, $\lambda$, causes instability regions are going to be wider than the smaller amounts of $\lambda \mathrm{s}$, which means the beam is going to lose its stability under external loading. The most instability region belongs to the $\lambda=1$ and uniform bending moment $(\psi=-1)$ load.(Figs. 4 and 5 ).

- According to Table 1 and Fig. 6 when the moment distribution factor, $\psi$, raises the instable region will be going to be narrower until being a line at limit case while asymmetric bending moment is applied. In other words the beam is fully stable if it is loaded asymmetrically.

- In general, decreasing the share of static buckling moment percentage, $\lambda$ and using higher values for moment distribution factor, $\psi$, in the $[-1,1]$ interval help to improve the dynamic stability of the beam.

\section{Appendix}

$$
\begin{aligned}
& A_{i j}=\int_{0}^{L} f_{i}^{\prime \prime}(x) f_{j}^{\prime \prime}(x) d x, \quad B_{i j}=\int_{0}^{L} g_{i}^{\prime \prime}(x) g_{j}^{\prime \prime}(x) d x \\
& C_{i j}=\int_{0}^{L} g_{i}^{\prime}(x) g_{j}^{\prime}(x) d x, \quad D_{i j}=-\int_{0}^{L}{ }^{0} M_{y}(x) f_{i}^{\prime \prime}(x) g_{j}(x) d x \\
& E_{i j}=\int_{0}^{L} f_{i}(x) f_{j}(x) d x, \quad E_{i j}^{*}=\int_{0}^{L} g_{i}(x) f_{j}(x) d x \\
& H_{i j}=\int_{0}^{L} g_{i}(x) g_{j}(x) d x, \quad H_{i j}^{*}=\int_{0}^{L} f_{i}(x) g_{j}(x) d x \\
& \mathbf{M}=\left[\begin{array}{cc}
\rho A \mathbf{E} & \rho A e \mathbf{H}^{*} \\
\rho A e \mathbf{E}^{*} & \rho I_{p} \mathbf{H}
\end{array}\right] \\
& \mathbf{K} e=\left[\begin{array}{cc}
E I_{z} \mathbf{A} & \mathbf{0} \\
\mathbf{0} & E I_{\omega} \mathbf{B}+G J \mathbf{C}
\end{array}\right] \\
& \mathbf{S}=\left[\begin{array}{ccc}
\mathbf{0} & \mathbf{D} \\
\mathbf{D}^{T} & \mathbf{0}
\end{array}\right]
\end{aligned}
$$

\section{References}

1 Dokumaci E, An exact solution for coupled bending and torsion vibrations of uniform beams having single cross-sectional symmetry, Journal of Sound and Vibration, 119(3), (1987), 443-449, DOI 10.1016/0022-460X(87)904081.

2 Miao S, Bioshop RED, Cannon SM, On coupled bending and torsional vibration of uniform beams, Journal of Sound and Vibration, 131(3), (1989), 457-464, DOI 10.1016/0022-460X(89)91005-5.

3 Pi Y-L, Bradford MA, Effects of approximations in analyses of beams of open thin-walled cross-section-Part I:Flexural-torsional stability, International Journal for Numerical Methods In Engineering, 51(7), (2001), 757772, DOI 10.1002/nme.155.abs.

4 Vörös GM, Buckling and free vibration analysis of stiffened panels, ThinWalled Structures, 47(4), (2009), 382-390, DOI 10.1016/j.tws.2008.09.002.

5 Vörös GM, On coupled bending-torsional vibrations of beams with initial loads, Mechanics Research Communications, 36(5), (2009), 603-611, DOI 10.1016/j.mechrescom.2009.01.006.

6 Talimian A, Vörös GM, 11th Hungarian Conference on Theoretical and Applied Mechanics, In:, HCTAM Conference Proceedings, 2011.

7 Faraday M, On a peculiar class of acoustical figures and on certain forms assumed by a group of particles upon vibratory elastic surfaces, Philosophical Transactions, 121, (1831), 299-340, DOI 10.1098/rstl.1831.0018.

8 Baliaev NM, Stability of prismatic rods subjected to variable longitudinal forces, Engineering Constructions and Structural Mechanics, (1924), 149167.

9 Bolotin VV, Weingarten VI, Trirogoff KN, Gallegos KD, The Dynamic Stability of Elastic systems, Aerospace corporation; El Segundo, California, 1962.

10 Brown JE, Hutt JM, Salma AE, Finite element solution to dynamic stability of bars, AIAA, 6(7), (1968), 1423-1425, DOI 10.2514/3.4779.

11 Thomas J, Abbas BAH, Dynamic Stability of Timoshenko Beams by Finite Element Method, Journal of Engineering for Industry, 98(4), (1976), 11451149, DOI 10.1115/1.3439069.

12 Snehasis G, Datta PK, Study of second regions of dynamic instability of a uniform beam, International Journal of Mechanical Education, 32(4), (2004), 292-299, DOI 10.7227/IJMEE.32.4.3.

13 Briseghella L, Majorana CE, Pellegrino C, Dynamic stability of elastic structures: a finite element approach, Computers and Structures, 69(1), (1998), 11-25, DOI 10.1016/S0045-7949(98)00084-4.

14 Kim M, Chang SP, Park HG, Spatial post buckling analysis of non symmetric thin-walled frames I: Theoretical considerations based on semi tangential property, Journal of Engineering Mechanics (ASCE), 127(8), (2001), 769778, DOI 10.1061/(ASCE)0733-9399(2001)127:8(769).

15 Wei-Chau Xie, Dynamic Stability of Structures, Cambridge University press, 2011. 